\title{
Lower Hybrid Wavepacket Stochasticity Revisited
}

\author{
V. Fuchs ${ }^{a}$, H. P. Laqua ${ }^{b}$, L. Krlín ${ }^{a}$, R. Pánek ${ }^{a}$, J. Preinhaelter ${ }^{a}$, J. Seidl ${ }^{a}$, J. Urban ${ }^{a}$ \\ ${ }^{a}$ Institute of Plasma Physics AS CR, v.v.i., Prague, CZ, Association EURATOM/IPP.CR \\ ${ }^{b}$ Max Planck Institute für Plasmaphysik, EURATOM Association, D-17491 Greifswald, German
}

\begin{abstract}
Analysis is presented in support of the explanation in Ref. [1] for the observation of relativistic electrons during Lower Hybrid (LH) operation in EC pre-heated plasma at the WEGA stellarator [1,2]. LH power from the WEGA TE11 circular waveguide, $9 \mathrm{~cm}$ diameter, un-phased, $2.45 \mathrm{GHz}$ antenna, is radiated into a $\mathrm{B} \cong 0.5 \mathrm{~T}, \overline{\mathrm{n}}_{\mathrm{e}} \cong 5 \times 10^{17} 1 / \mathrm{m}^{3}$ plasma at $\mathrm{T}_{\mathrm{e}} \cong 10 \mathrm{eV}$ bulk temperature wth a EC generated $50 \mathrm{keV}$ component [1]. The fast electrons cycle around flux or drift surfaces essentially without collisions and repeatedly interact with the rf field close to the antenna mouth, gaining energy in the process. Our antenna calculations reveal a standing electric field pattern at the antenna mouth, with which we formulate the electron dynamics via a relativistic Hamiltonian. A simple approximation of the equations of motion leads to a relativistic generalization of the areapreserving Fermi-Ulam (F-U) map [3], allowing phase-space global stochasticity analysis. At typical WEGA plasma and antenna conditions, the F-U model predicts an LH driven current of about $230 \mathrm{~A}$, at about $225 \mathrm{~W}$ of dissipated power, in good agreement with the measurements and analysis reported in [1].
\end{abstract}

Keywords: stellarator, lower hybrid, current drive, Hamiltonian, electron, stochastic.

PACS: 52.55.Hc, 52.50.-b, 52.20.Dq, 52.50.Gj, 52.50.Sw, 05.40.-a

\section{INTRODUCTION}

This work's motivation is driven by the wish to provide an alternative explanation to the analysis of the observation of relativistic $\mathrm{MeV}$ electrons during Lower Hybrid (LH) operation in EC pre-heated plasma at the WEGA stellarator, reported in Ref [1]. To do so, we, first, extend our previous work [4-8] on electron phase space stochasticity caused by electrons repeatedly passing through a spatially localized travelling lower hybrid (LH) wave to the case of electrons passing through a standing wave. We note that Ref. [8] presented a relativistic extension of the exact nonlinear results of [5-7]. Second, we show that the standing wave interaction can be approximated by the "simplified" version of the Fermi-Ulam map [3], which we here generalize to relativistic electron velocities.

Calculations of the electromagnetic power radiating at $2.45 \mathrm{GHz}$ into a $\mathrm{T}_{\mathrm{e}} \cong 10 \mathrm{eV}$, line average $\overline{\mathrm{n}}_{\mathrm{e}} \cong 5 \times 10^{17} 1 / \mathrm{m}^{3}$, $\mathrm{B} \cong 0.5 \mathrm{~T}$, edge plasma by the WEGA TE11 circular waveguide, $9 \mathrm{~cm}$ diameter, un-phased antenna, indicate that at the given conditions the electric field is polarized predominantly in the $\mathrm{z}$ (toroidal) direction; with $40 \%$ of the power reflected. Most of the transmitted power ends up in non-propagating eigenmodes, only 10\% propagates in opposite directions along resonance cones, which due to the lack of phasing also forms a standing wave pattern at the antenna mouth, where the $\sim \mathrm{MeV}$ electrons were observed [1]. The standing wave field can be described by a relativistic nonconservative and non-autonomous Hamiltonian, which, in contrast to the case of a travelling plane wave with a rectangular envelope [5-8], is not conserved.

Fast electrons $(\geq 50 \mathrm{keV})$ cycle essentially without collisions around a flux or drift surface (as indicated below in FIG.1 of Ref [1]) in the low density plasma with an EC generated $50 \mathrm{keV}$ component, and repeatedly interact with the rf field close to the antenna mouth. The electrons are not bound to a constant energy surface in phase space, so they can, in principle, gain energy, but for coherent phases between interaction events the energy is limited by a global stochasticity bound which we shall determine here. Specifically, we show that a simple approximation of the equations of motion associated with the Hamiltonian (1) below leads to a relativistic version of the area-preserving F-U map. The present form of the relativistic F-U map is distinct from a previous relativistic F-U model - the gravitational bouncer model [9] - in which the particle energy can increase indefinitely even if the phase delay between interaction events is not random. From numerical and period-one fixed point stability analysis [3], applied here to the F-U relativistic case, we find that the electron energy U does not exceed at WEGA conditions about 300 $\mathrm{keV}$, unless the phases are random.

References [1,2] report that at energies above $200 \mathrm{keV}$, the electron confinement in WEGA becomes highly asymmetric with electrons in one direction along drift surfaces suffering loss, so a non-inductive current is a fortiori generated. First moments of the electron distribution obtained from the iterated F-U map give an LH current density 
of about $7 \mathrm{kA} / \mathrm{m}^{2}$, an LH driven current $\cong 230 \mathrm{~A}$, and dissipated LH power $\cong 225 \mathrm{~W}$. This compares favorably with results of Ref. [1].

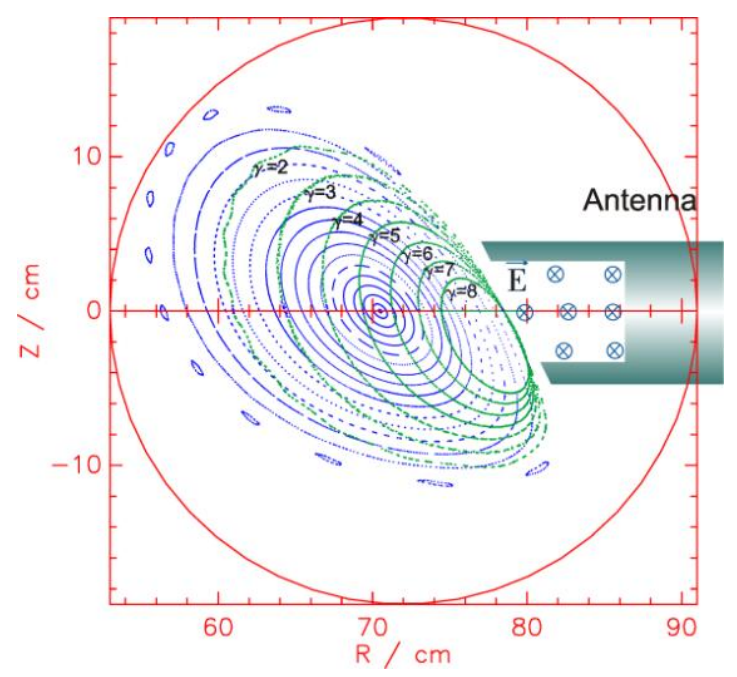

FIGURE 1. Flux surfaces (blue) and particle drift surfaces (green) for different relativistic $\gamma$-factors. On the left there is a simplified sketch of the antenna field. This is a poloidal cut of a toroidal configuration

\section{THE RELATIVISTIC FERMI-ULAM MAP}

The standing wave relativistic Hamiltonian with canonical momentum $\mathrm{p}$ and coordinate $\mathrm{z}$ is

$$
H=m_{e} c^{2}(\gamma-1)+\Phi(z) \sin (\omega t), \quad \Phi(z)=\$ E_{0} / \omega k-\sin (k z) .
$$

The corresponding equations of motion

$$
\frac{d p}{d t}=-\frac{\partial H}{\partial z}=\sin (\omega t) \frac{\partial \Phi}{\partial z} ; \quad \frac{d z}{d t}=\frac{\partial H}{\partial p}=\frac{p}{m_{e} \gamma} ; \quad \gamma=\sqrt{1+\left(\frac{p}{c m_{e}}\right)^{2}}
$$

describe electrons orbiting around flux (or drift) surfaces and exchanging momentum with the field when passing by the antenna. Denote by $\mathrm{z}_{0}=0$ the position of the antenna, by $\mathrm{t}_{0}$ the time during an orbit at which interaction occurs, and by $\mathrm{L}$ the electron orbit length. Integrating Eqs (1) for one orbit we obtain:

$$
\begin{aligned}
& p_{1}-p_{0}=m_{e} v_{q} \int \sin (\omega t) \cos [k z(t)] \delta\left(t-t_{0}\right) d t=m_{e} v_{q} \sin \left(\omega t_{0}\right) \cos \left(k z_{0}\right) \\
& z_{1}-z_{0} \equiv L=\frac{p_{1}}{m_{e} \gamma_{1}}\left(t_{1}-t_{0}\right) ; \quad v_{q}=\frac{e E_{0}}{\omega m_{e}}
\end{aligned}
$$

With $\mathrm{z}_{0}=0$, phase $\psi=\omega \mathrm{t}$, and normalized momentum $\mathrm{u}=\mathrm{p} /\left(\mathrm{m}_{\mathrm{e}} \mathrm{v}_{\mathrm{q}}\right)$, Eqs (3) give the relativistic form of the F-U map

$$
u_{n+1}=u_{n}+\sin \left(\psi_{n}\right) ; \quad \psi_{n+1}=\psi_{n}+\frac{2 \pi M \gamma_{n+1}}{u_{n+1}} ; \quad M=\frac{L f}{v_{q}}
$$

where $\mathrm{E}_{0} \cong 0.56 \mathrm{kV} / \mathrm{cm}$ is the lower hybrid $\mathrm{E}_{\mathrm{z}}$-field amplitude and $\omega=2 \pi \mathrm{f}$.

The F-U map is a resonant system with the principal, so-called period 1 resonances [3], occurring when the electron orbit time $L / v$ equals an integer multiple of the field period $1 / f$, i.e. when $L / v \equiv \mathrm{Lm}_{\mathrm{e}} \gamma / \mathrm{p}=\mathrm{n} / \mathrm{f}, \mathrm{n}=1,2,3, \ldots$ In the present relativistic version we need to formulate the resonance condition in the same frame of reference for the two oscillating processes. So, for example, in the antenna rest frame of reference all processes experienced by the moving electron are perceived by an observer at rest as slowed down by the Lorentz factor $\gamma$. Hence the momentum space resonance condition becomes simply $\mathrm{Lm}_{\mathrm{e}} / \mathrm{p}=\mathrm{n} / \mathrm{f}$, or $\mathrm{M} / \mathrm{u}=\mathrm{n}$ in normalized form.

\section{Global Stochasticity Boundary from Period 1 Momentum}


With the time dilation of the electron orbit taken into account, mapping (4) becomes

$$
u_{n+1}=u_{n}+\sin \left(\psi_{n}\right) ; \quad \psi_{n+1}=\psi_{n}+2 \pi M / u_{n+1}
$$

One of the standard methods for finding phase space stochasticity domains of (5) is to locally represent the F-U map by the "Standard" map whose global stochasticity properties are well known [3]. We thus linearize (5) around the period 1 momentum $\mathrm{u}_{1}=\mathrm{M} / \mathrm{n}$ with $\mathrm{u}=\mathrm{u}_{1}+\Delta \mathrm{u}$, shift the phase $\psi=\Theta-\pi / 2$, and introduce a new action variable $\mathrm{I}=\mathrm{K} \Delta \mathrm{u}$, where $\mathrm{K}=2 \pi \mathrm{M} /\left(\mathrm{u}_{1}\right)^{2}$ is the stochasticity parameter. The map (5) is thus locally represented by the "Standard" map

$$
I_{n+1}=I_{n}+K \sin \left(\Theta_{n}\right) ; \quad \Theta_{n+1}=\Theta_{n}+I_{n+1}
$$

which exhibits global stochasticity when $\mathrm{K} \geq 1$, i.e. when $2 \pi \mathrm{M} /\left(\mathrm{u}_{1}\right)^{2} \geq 1$. The global stochasticity momentum threshold is therefore $p_{1} \leq m_{e} \sqrt{ } \omega_{L H} L_{q}$, with the corresponding resonance number $n$ satisfying $n \geq \sqrt{M} / 2 \pi$. This last inequality expresses the stochasticity threshold in terms of the Chirikov criterion which essentially states that when the excitation $\left(E_{0}\right)$ is not strong enough, then the lower order resonances run out of overlap.

\section{APPLICATION TO WEGA}

We now apply results of the preceding section to the WEGA LH operation conditions of Ref. [1]: electron orbit mean radius at $\gamma=2$ from FIG. 1, $\mathrm{R} \cong 0.4 \mathrm{~m}$, safety factor $\mathrm{q} \cong 5$, orbit length $\mathrm{L} \cong 12.5 \mathrm{~m}, \mathrm{v}_{\mathrm{q}} \cong 6.4 \times 10^{5} \mathrm{~m} / \mathrm{s}$, stochasticity parameter $\mathrm{M}=4.78 \times 10^{4}$, the period 1 momentum threshold from the condition $\mathrm{K}=1, \mathrm{p}_{1}=3.2 \times 10^{-22} \mathrm{~kg} \mathrm{~m} / \mathrm{s}$, and threshold resonance mode number $n=87$. The corresponding energy stochasticity threshold $U_{1}=512\left(\gamma_{1}-1\right)$, with $\gamma_{1}=\sqrt{ } 1+\left(\mathrm{p}_{1} / \mathrm{c} \mathrm{m}_{\mathrm{e}}\right)^{2}$ is therefore $\mathrm{U}_{1}=276 \mathrm{keV}$, dismally short of the expected $\mathrm{MeV}$ range. This indicates that the phases between interaction events are random, as indicated in Ref. [1]. To confirm this, we carry out some simulations at the given parameters with the F-U map (4) and numerical integration of Eqs (2). Results are shown in FIGS 2 and 3. First, in FIG. 2 we show results for correlated phases.
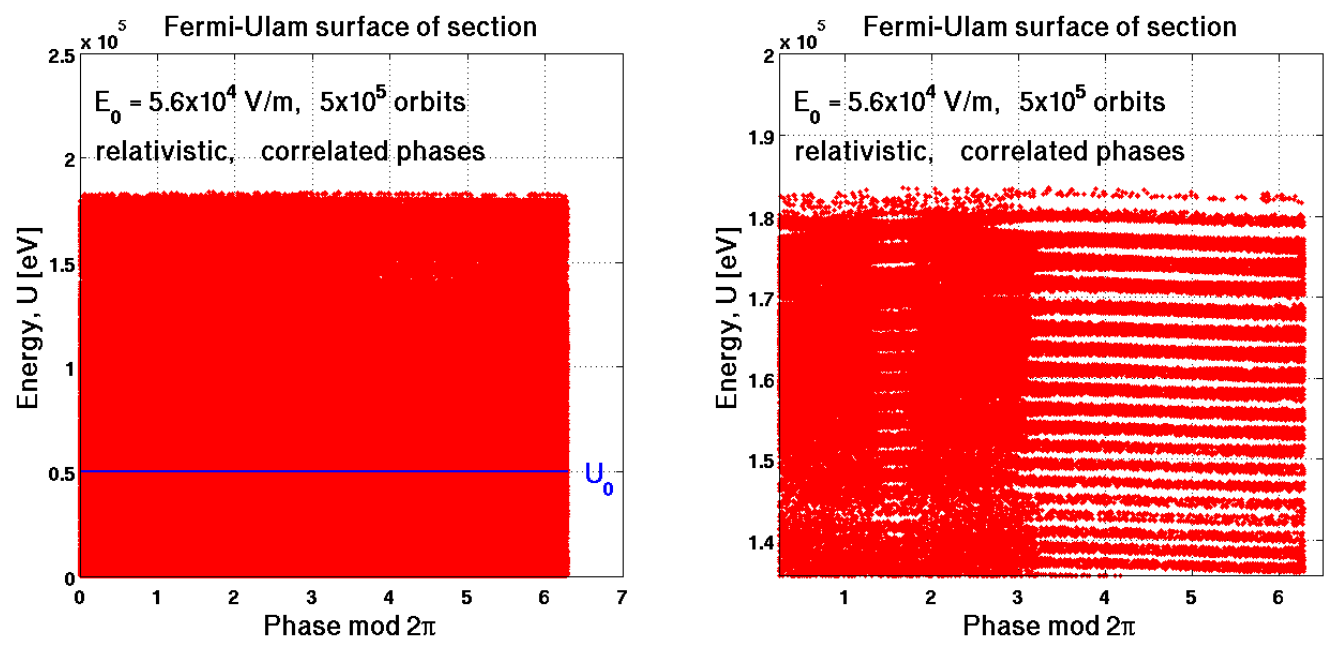

FIGURE 2. Fermi-Ulam map (4) surface of section, energy versus phase, at WEGA operating conditions of Ref.[1]. Phases between interaction events are here assumed correlated. The lowest-order resonances are evident in the detail of the stochasticity boundary shown on the right. $\mathrm{U}_{0}$ indicates the energy initial condition.

A different picture emerges with random phases, as shown in FIG. 3 below. First, FIG. 3a indicates that the stochastic barrier of FIG. 2 is destroyed. The electron energy now increases with the number of orbits. The same tendency follows from the diffusion coefficient $D_{k}$ of FIG. $3 b$, which indicates saturation as function of electron initial energy $E_{\text {in }}$. The final energy $E_{\text {out }}$ is obtained as in Ref. 1, i.e. by numerical integration through the field with a Gaussian envelope. The resulting $D_{k}$ is an ensemble average for one orbit over $10^{7}$ electrons.

\section{CONCLUSION}


To summarize, there are two main factors allowing the generation of $\mathrm{MeV}$ electrons and current drive during LH operation with an un-phased antenna at WEGA. First, the $50 \mathrm{keV}$ electrons generated during EC pre-heating are sufficiently collisionless in order to cycle around drift surfaces and thereby to gain energy by repeated interaction with the field at the antenna mouth. In so doing the electrons become even less collisional. This is a typical runaway situation - a critical velocity for runaway occurs when the fast electron mean-free-path is larger than the total orbiting path. Second, motion on the drift surfaces is asymmetric, such that electrons in one direction are not confined and in consequence of that a current is generated.

Thus, finally, the iterated F-U map yields global quantities of interest such as the driven current density $\mathrm{J}=\mathrm{e}$ $\mathrm{n}_{\text {ehot } \mathrm{e}}\langle\mathrm{v}\rangle_{\text {bounce }}\left[\mathrm{A} / \mathrm{m}^{2}\right]$ and the dissipated power density $\mathrm{Q}=\mathrm{J}\langle\mathrm{dp} / \mathrm{dt}\rangle_{\text {bounce }}\left[\mathrm{W} / \mathrm{m}^{3}\right]$. For the sake of definiteness,

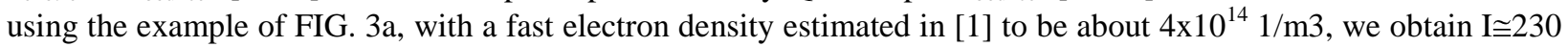
$\mathrm{A}$ and dissipated power $\mathrm{P} \cong 225 \mathrm{~W}$. This gives a promising $\mathrm{LH}$ current drive efficiency $\mathrm{I} / \mathrm{P}_{\mathrm{abs}}$ of order 1 .
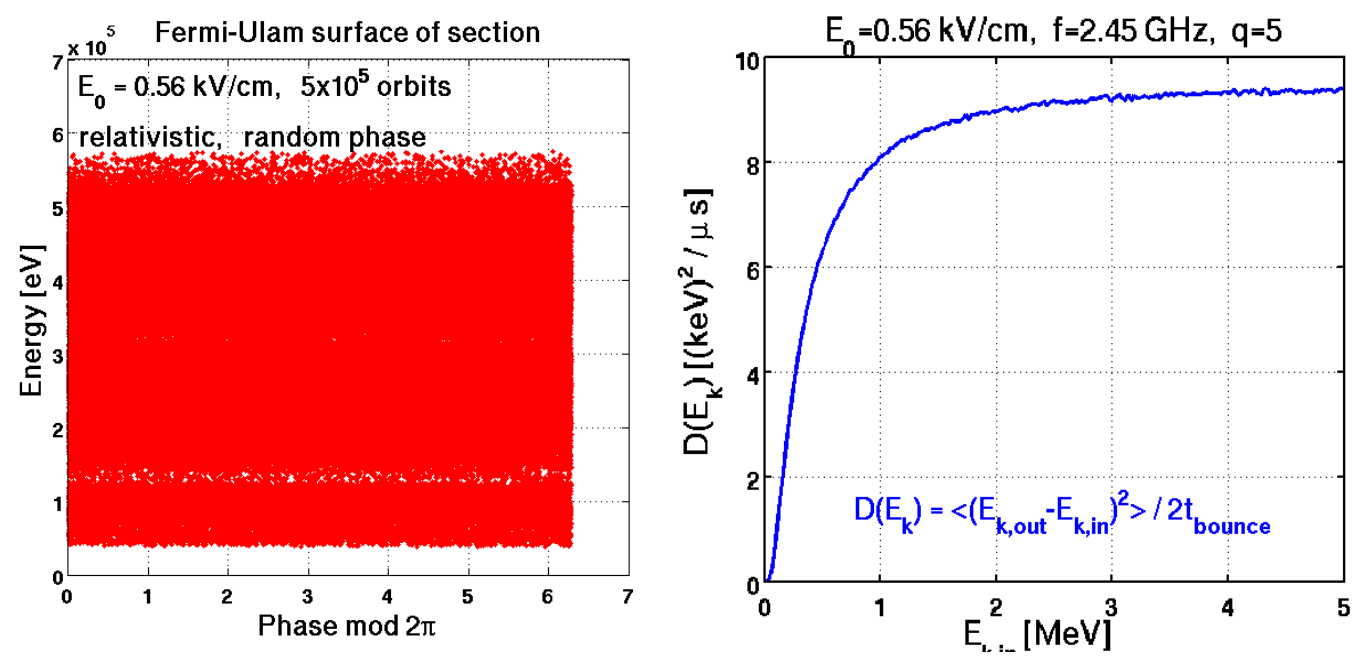

FIGURE 3 a) Fermi-Ulam map surface of section, energy versus phase, at WEGA operating conditions of Ref.[1] for random phases between interaction events.. b) Diffusion coefficient from numerical integration of Eqs. (2) as function of electron initial energy. An ensemble of $10^{7}$ electrons is distinguished by initial random phases.

\section{ACKNOWLEDGMENTS}

\section{This work was partly supported by MSMT Project LM2011021.}

\section{REFERENCES}

1. H. P. Laqua, E. Chlechowitz, M. Otte and T. Stange, "Stochastic Landau-Like Acceleration of Relativistic Electrons and Plasma Heating and Current Drive at the WEGA Stellarator", submitted to Physical Review Letters.

2. M. Otte, H. P. Laqua, E. Chlechowitz, S. Marsen, J. Preinhaelter, T. Stange, A. Rodatos, J. Urban, D. Zhang, "Overview of Recent Results From the WEGA Stellarator",Nukleonika,57,:171-175, (2012).

3. A. J. Lichtenberg, M. A. Lieberman, "Regular and Stochastic Motion", Springer-Verlag, New York, (1983).

4. V. Fuchs, V. Krapchev, A. Ram and A. Bers, "Diffusion of Electrons by Coherent Wavepackets", Physica, 14D, 141-160, (1985).

5. P. Pavlo and L. Krlin, "Limits of applicability of the quasilinear approximation to the lower hybrid-plasma interaction", Plasma Phys. Control. Fusion, 41, 541-550, (1999).

6. R. Panek, PhD Thesis, "Nonlinear and Stochastic Interaction of Waves with Plasma"Charles University, Prague (2002).

7. L. Krlín, P. Pavlo, R. Pánek, R. Klíma and V. Petržílka, "Nonlinear Effects in LH Wave-Plasma Interaction", Plasma Phys. Control. Fusion, 44, 159-170, (2002).

8. J. Seidl, Master Thesis: "Anomalous diffusion of electrons interacting with a lower hybrid wave in a tokamak", Charles University, Prague (2006).

9. L. D. Pustylnikov, "New Mechanism of Particle Acceleration and Relativistic Analogue of the Fermi-Ulam Model", Teoretical and Mathematical Physics, 77, 1110-1115, (1988). 